\title{
A Comparison of Three Stator Resistance Estimation Methods for a Permanent Magnet Motor
}

\author{
Alia R. Strandt, Andrew P. Strandt, Susan C. Schneider, Edwin E. Yaz \\ Marquette University \\ 1250 W. Wisconsin Ave., Milwaukee, WI, USA \\ alia.strandt@mu.edu; andrew.strandt@mu.edu; susan.schneider@mu.edu; edwin.yaz@mu.edu
}

\begin{abstract}
In this work, three adaptive estimation methods are considered for the identification of the stator winding resistance of an interior permanent magnet motor. The layout of the magnets in the motor under consideration produces a trapezoidal back electromotive force (emf), which is more challenging for the estimators due to the introduction of higher order harmonics. The three estimation techniques are compared in terms of accuracy in estimating the true parameter value. The multiple model estimation (MME) algorithm utilizing Kalman filters provides the most accurate estimate with the least computational complexity while the additional complexity of the extended Kalman filter (EKF) and the fading memory extended Kalman filter (FM-EKF) results in a poor estimate of the parameter.
\end{abstract}

Keywords: parameter identification, PM motors, motor-drive systems

\section{Introduction}

In the field of motor-drives, most control techniques still utilize physical model-based methods that rely on the knowledge of parameter values [1]. Using parameter-based models facilitates fast and accurate control of the motor-drive, but introduces the need for fast and accurate parameter identification for optimal control results, especially if the parameters are varying with operating conditions and require occasional re-identification [2]. Stator resistance is a parameter especially sensitive to operating conditions, necessitating fast and accurate parameter estimation [3].

Several investigations have attempted to address the stator winding resistance estimation problem. In [4], the authors propose a method to estimate the stator parameters, including stator resistance, using only voltage and current measurements without rotor operation information. They also analyze the sensitivity of the stator resistor estimation to errors in the estimates of other motor parameters. In [5], the authors demonstrate how error in the stator resistance estimation detunes the control performance. These authors include an additional PI controller to act on the error in the angle between the stator flux and stator current to estimate stator resistance during operation when performance degrades. Other methods for estimating or compensating for stator resistance error are similar to the method proposed in [6]. Here, a stepwise direct-current excitation is used and the stator current periodically sampled and post-processed. The authors show how the method tracks stator resistance estimation changes well, though with a 10 second time delay, and they demonstrate how to track stator winding temperature changes using the stator resistance estimate and an external thermometer.

This work extends [7] by comparing three adaptive estimation techniques when applied to the stator winding resistance identification problem. Section 2 presents the permanent magnet (PM) motor models used in this work. The three adaptive estimation techniques, the multiple model estimation (MME) algorithm utilizing a bank of Kalman filters, the extended Kalman filter (EKF), and the fading memory extended Kalman filter (FM-EKF), are discussed in Section 3. The simulation and the estimation results for the case study motor are presented in Section 4. Finally, Section 5 summarizes the results and provides conclusions.

\section{The Permanent Magnet Synchronous Motor Model}

The continuous time permanent magnet motor model in the $a b c$ coordinate system using phase currents as state variables [8] is used to generate the motor measurement data.

$$
\dot{i}_{a b c}=-L^{-1}\left(R_{s}+\dot{L}\right) i_{a b c}+L^{-1}\left(v_{a b c}-e_{a b c}\right)
$$


Here, $i_{a b c}=\left[\begin{array}{lll}i_{a} & i_{b} & i_{c}\end{array}\right]^{T}$ are the phase currents in Amperes (A), $v_{a b c}=\left[\begin{array}{lll}v_{a} & v_{b} & v_{c}\end{array}\right]^{T}$ are the terminal phase voltages in Volts $(\mathrm{V}), e_{a b c}=\left[\begin{array}{lll}e_{a} & e_{b} & e_{c}\end{array}\right]^{T}$ are the voltages induced by the back electromotive force (emf) in Volts, $R_{s}$ is the diagonal matrix consisting of the stator resistance $r_{s}$ in Ohms $(\Omega), L$ is the matrix of time-varying self and mutual inductances of the stator windings in Henries $(\mathrm{H})$, and $\dot{L}$ is the time derivative of the $L$ matrix which is calculated elementby-element.

Note that (1) is a time-varying model. In practice, it is advantageous to transform the model for estimation and control purposes in such a way that it becomes time invariant. The dq0 coordinate transformation projects the motor currents and voltages into a reference frame that rotates at the synchronous speed of the motor, reducing sinusoids at synchronous frequency to dc quantities determined by magnitudes and phase angles. The amplitude invariant dq0 transformation $T_{d q 0}$ and its inverse $T_{d q 0}^{-1}$ are presented in (2) and (3).

$$
\begin{gathered}
T_{d q 0}=\frac{2}{3}\left[\begin{array}{ccc}
\cos (\sigma) & \cos \left(\sigma-\frac{2 \pi}{3}\right) & \cos \left(\sigma-\frac{4 \pi}{3}\right) \\
-\sin (\sigma) & -\sin \left(\sigma-\frac{2 \pi}{3}\right) & -\sin \left(\sigma-\frac{4 \pi}{3}\right) \\
\frac{1}{2} & \frac{1}{2} & \frac{1}{2}
\end{array}\right] \\
T_{d q 0}^{-1}=\left[\begin{array}{ccc}
\cos (\sigma) & -\sin (\sigma) & 1 \\
\cos \left(\sigma-\frac{2 \pi}{3}\right) & -\sin \left(\sigma-\frac{2 \pi}{3}\right) & 1 \\
\cos \left(\sigma-\frac{4 \pi}{3}\right) & -\sin \left(\sigma-\frac{4 \pi}{3}\right) & 1
\end{array}\right]
\end{gathered}
$$

Here, $\sigma$ is the electrical position of the rotor in radians. When the rotor speed is constant, this becomes $\sigma=\omega_{e} t$, where $\omega_{e}$ is the electrical rotational speed of the motor in electrical radians per second, and $t$ is the time instant in seconds.

Transforming (1) using (2) results in the $d q 0$ coordinate motor model (4) [9]

$$
\left[\begin{array}{l}
\dot{i}_{d} \\
\dot{i}_{q} \\
\dot{i}_{0}
\end{array}\right]=\left[\begin{array}{ccc}
-\frac{r_{s}}{L_{d d}} & \frac{\omega_{e} L_{q q}}{L_{d d}} & 0 \\
-\frac{\omega_{e} L_{d d}}{L_{q q}} & -\frac{r_{s}}{L_{q q}} & 0 \\
0 & 0 & -\frac{r_{s}}{L_{00}}
\end{array}\right]\left[\begin{array}{c}
i_{d} \\
i_{q} \\
i_{0}
\end{array}\right]+\left[\begin{array}{ccc}
\frac{1}{L_{d d}} & 0 & 0 \\
0 & \frac{1}{L_{q q}} & 0 \\
0 & 0 & \frac{1}{L_{00}}
\end{array}\right]\left[\begin{array}{l}
v_{d}-e_{d} \\
v_{q}-e_{q} \\
v_{0}-e_{0}
\end{array}\right]
$$

where $i_{d q 0}$ is the vector of $d q 0$ coordinate phase currents, $v_{d q 0}$ is the vector of $d q 0$ coordinate terminal phase voltages, and $e_{d q 0}$ is the vector of $d q 0$ coordinate voltages induced by the motor back emf. Additionally, $L_{d d}, L_{q q}$, and $L_{00}$ are the selfinductances of the stator projected into $d q 0$ coordinates. Note that this model is linear and time invariant when $\omega_{e}$ is constant.

The measurements of the system are the phase currents in $a b c$ coordinates. The inverse $d q 0$ transform in (3) is used to relate the dq0 currents to the measured values. Thus, the measurement equation is

$$
\left[\begin{array}{l}
i_{a} \\
i_{b} \\
i_{c}
\end{array}\right]=\left[\begin{array}{ccc}
\cos (\sigma) & -\sin (\sigma) & 1 \\
\cos \left(\sigma-\frac{2 \pi}{3}\right) & -\sin \left(\sigma-\frac{2 \pi}{3}\right) & 1 \\
\cos \left(\sigma-\frac{4 \pi}{3}\right) & -\sin \left(\sigma-\frac{4 \pi}{3}\right) & 1
\end{array}\right]\left[\begin{array}{l}
i_{d} \\
i_{q} \\
i_{0}
\end{array}\right]
$$

Note that neither system noise nor measurement noise is modeled in this work.

It is often useful to discretize continuous time systems when the estimator is implemented using a microcontroller. Implicit discretization methods, such as the trapezoidal method, generally are more accurate and numerically stable than explicit discretization methods, such as the Forward Euler method. However, it can be challenging to return the system to state space form, particularly for nonlinear systems. In the case of a linear time invariant (LTI) system, such as (4) during steady state operation, the trapezoidal discretization method can be written for LTI systems as

$$
\begin{aligned}
& x_{k+1}=A x_{k}+B u_{k} \\
& A=\left(I-0.5 T_{s} A_{c}\right)^{-1}\left(I+0.5 T_{s} A_{c}\right) \\
& B=0.5 T_{s}\left(I_{3}-0.5 T_{s} A_{c}\right)^{-1} B_{c}
\end{aligned}
$$


where $A_{c}$ is the continuous time $A$ matrix, $B_{c}$ is the continuous time $B$ matrix, $T_{s}$ is the sampling period in seconds, and $I$ is the identity matrix of appropriate dimension. Since parameter identification with the EKF or the FM-EKF results in a nonlinear problem, (4) is discretized using the Forward Euler method:

$$
\left[\begin{array}{l}
i_{d, k+1} \\
i_{q, k+1} \\
i_{0, k+1} \\
r_{s, k+1}
\end{array}\right]=\left[\begin{array}{c}
i_{d, k}-T_{s} \frac{r_{s, k}}{L_{d d}} i_{d, k}+T_{s} \frac{\omega_{e} L_{q q}}{L_{d d}} i_{q, k} \\
i_{q, k}-T_{s} \frac{\omega_{e} L_{d d}}{L_{q q}} i_{d, k}-T_{s} \frac{r_{s, k}}{L_{q q}} i_{q, k} \\
i_{0, k}-T_{s} \frac{r_{s, k}}{L_{00}} i_{0, k} \\
r_{s, k}
\end{array}\right]+\left[\begin{array}{c}
T_{s} \frac{1}{L_{d d}}\left(v_{d, k}-e_{d, k}\right) \\
T_{s} \frac{1}{L_{q q}}\left(v_{q, k}-e_{q, k}\right) \\
T_{s} \frac{1}{L_{00}}\left(v_{0, k}-e_{0, k}\right) \\
0
\end{array}\right]
$$

\section{The Estimators}

\subsection{Multiple Model Estimation (MME) utilizing Kalman Filters}

The multiple model estimation algorithm estimates an unknown system parameter such as $r_{S}$ by creating a set $R$ of $N$ hypotheses. One estimator is designed around each hypothesis, and the posterior probability that the $i^{t h}$ hypothesis $r_{s, i}$ is closest to the true parameter value is calculated using Bayes' rule $(10)$. Here, $p(*)$ represents a probability density function, $y_{k}$ is the measurement at time $k$, and $Y_{k}$ is the set of all measurements through time $k$.

$$
p\left(r_{s, i} \mid Y_{k}\right)=\frac{p\left(y_{k} \mid Y_{k-1}, r_{s, i}\right) p\left(r_{s, i} \mid Y_{k-1}\right)}{\sum_{m=1}^{N} p\left(y_{k} \mid Y_{k-1}, r_{s, m}\right) p\left(r_{s, m} \mid Y_{k-1}\right)}
$$

Fig. 1 outlines this process. A more detailed explanation of the MME algorithm and its application to stator resistance estimation can be found in [7]. Since the MME algorithm preserves the linearity of the system, it is possible to discretize the $d q 0$ coordinate model in (4) using the trapezoidal method. The hypothesis set $R=\{0.2,0.3,0.4,0.5,0.6\}$ consists of five possible values for the stator resistance, each of which is equally likely to be closest to the true stator resistance. In practice, if a certain nominal value is more likely than others, the initial posterior probabilities can be adjusted to reflect this.

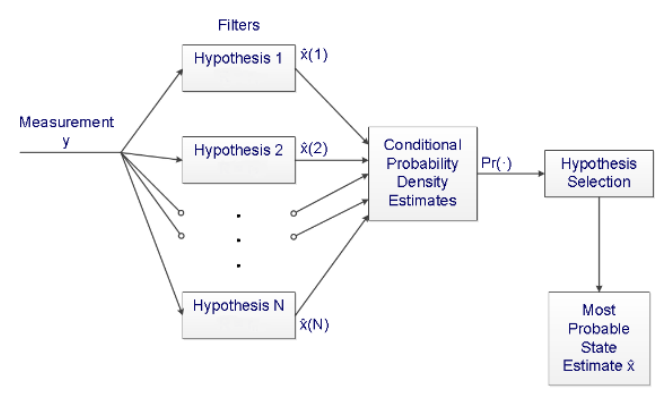

Fig. 1: Block diagram of the multiple model estimation algorithm [10].

\subsection{The Extended Kalman Filter (EKF)}

Consider a nonlinear time varying stochastic system of the form

$$
\begin{gathered}
x_{k+1}=f\left(x_{k}, u_{k}\right)+F_{k} v_{k} \\
y_{k}=h\left(x_{k}, u_{k}\right)+G_{k} w_{k}
\end{gathered}
$$

where $x_{k}$ is the $n \times 1$ state vector, $u_{k}$ is the $m \times 1$ input vector, and $y_{k}$ is the $p \times 1$ measurement vector. The $n \times 1$ system noise vector $v_{k}$, the $p \times 1$ measurement noise vector $w_{k}$, and the initial state value $x_{0}$ are independent white random variables with Gaussian densities as in (18) of the Appendix. The extended Kalman filter for model (11) and (18) can be expressed as in (12) below. See the Appendix for the complete set of equations [11]. The innovations term $\tilde{y}_{k}$ is given in (13).

$$
\begin{gathered}
\hat{x}_{k+1}=f\left(\hat{x}_{k}, u_{k}\right)+K_{k} \tilde{y}_{k} \\
\tilde{y}_{k}=y_{k}-\hat{y}_{k}=y_{k}-h\left(\hat{x}_{k}, u_{k}\right)
\end{gathered}
$$

To use the Kalman filter gain and covariance equations found in (20) and (21) of the Appendix, the nonlinear system is linearized around the current estimate $\hat{x}_{k}$. Thus, $A_{k}$ and $C_{k}$ are found from the Jacobians of the nonlinear system 


$$
A_{k}=\left.\frac{\partial f}{\partial x}\right|_{x=\hat{x}, u=u_{k}} \quad C_{k}=\left.\frac{\partial h}{\partial x}\right|_{x=\hat{x}, u=u_{k}}
$$

In the parameter identification problem, the unknown parameter is appended to the state vector, and the dynamic model for this parameter is appended to the system vector. In the case of a constant parameter such as the stator resistance, the parameter model is of the form of (15). In this work, the EKF is designed using the $d q 0$ coordinate motor model discretized using the Forward Euler method as found in (9) and is initialized with a value of $\hat{r}_{s, 0}=0.3 \Omega$.

$$
r_{s, k+1}=r_{s, k}
$$

\subsection{The Fading Memory Extended Kalman Filter (FM-EKF)}

Several techniques have been developed that can improve the performance of the Kalman filter and the EKF [12]. One such technique involves weighting recent measurements more heavily than older measurements. This modification is known as the fading memory extended Kalman filter [12]. It is particularly useful when there may be a mismatch between the system and the model because it prioritizes the recent measurement information over the expected system dynamics. The estimator, the Kalman gain, and the Jacobians remain the same as in the standard formulation of the EKF. The covariance equation includes a forgetting factor $\alpha$ that is slightly larger than 1, which prevents $P_{k}$ from becoming too small. The increase in $P_{k}$ reflects a lack of confidence in the estimate, which results in a larger estimator gain. Thus, the innovations will have a larger effect on the estimate at each time step. The modified covariance equation is given by

$$
P_{k+1}=\alpha^{2} A_{k} P_{k} A_{k}^{T}+F_{k} V_{k} F_{k}^{T}-\alpha^{2} A_{k} P_{k} C_{k}^{T}\left(C_{k} P_{k} C_{k}^{T}+G_{k} W_{k} G_{k}^{T}\right)^{-1} C_{k} P_{k} A_{k}^{T}
$$

Note that if the value of $\alpha$ is too large, the system model is completely neglected, and the estimator will not converge; $\alpha=1.01$ is commonly selected. As with the EKF, the FM-EKF is designed using the model in (9) and is initialized with a value of $\hat{r}_{s, 0}=0.3 \Omega$. In addition, the value of $\alpha$ is fixed to 1.01 .

\section{Simulation Results}

\subsection{The Case Study $3.5 \mathrm{hp}$ Motor}

Table 1 presents the ratings of the case study motor. All motor parameters were previously calculated using Finite Element Analysis. In particular, the resistance of the stator resistance is $r_{s}=0.49 \Omega$, and the voltages induced by the back emf contain significant odd harmonics. This results in a trapezoidal waveform as seen in Fig. 2.

The sinusoidal terminal excitation is applied in such a way that the ratio of the voltage in Volts and the frequency in Hertz (V/f) remains constant. This control maintains constant torque across different motor speeds, assuming the voltage drop over the stator windings is small compared to the applied voltage. Three operating speeds are considered using constant voltage to frequency control: rated speed, one-half rated speed, and one-quarter rated speed. For the purpose of simulation, all input and output data are generated using the continuous time $a b c$ coordinate model of the motor in (1). The differential equations are solved using the stiff solver ode $23 \mathrm{~s}$ in MATLAB. It is assumed that the drive uses a constant sampling period of $T_{s}=200 \mu \mathrm{s}$. Thus, as the motor operating speed decreases, the number of samples per electrical cycle increases.

Table 1: Ratings of the Case Study Motor.

\begin{tabular}{|l|l|}
\hline Rated Power & $3.5 \mathrm{hp}$ \\
\hline Rated Speed & $3450 \mathrm{r} / \mathrm{min}$ \\
\hline Rated Torque & $7.25 \mathrm{Nm}$ \\
\hline Rated Current & $10 \mathrm{~A}$ \\
\hline Rated Line-to-Line Voltage & $240 \mathrm{~V}$ \\
\hline
\end{tabular}




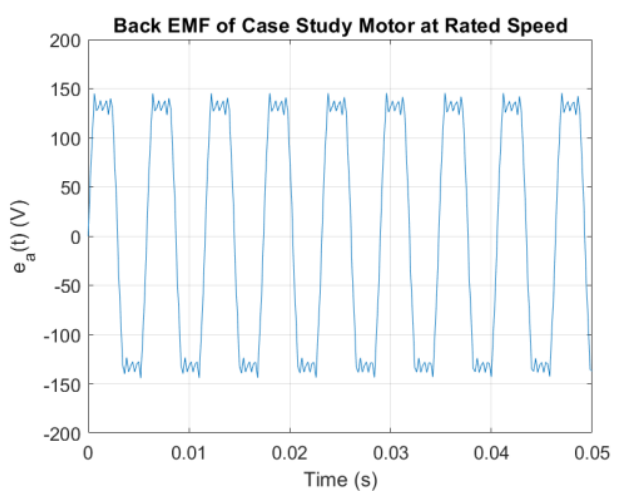

Fig. 2: Phase $a$ open circuit back emf of the motor at rated speed. The back emf of phase $b$ and phase $c$ are phase shifted $\pm 120^{\circ}$ from phase $a$. The waveform is trapezoidal.

\subsection{Results at Rated Speed}

In Fig. 3, the posterior probabilities of the five hypotheses of the MME algorithm are presented as a function of time. The probability curves corresponding to $R_{1}, R_{2}, R_{3}$, and $R_{5}$ approach 0 , while the probability curve corresponding to $R_{4}$ approaches 1 . This indicates that the hypothesis $R_{4}=0.5 \Omega$ is the closest to the correct value of $0.49 \Omega$. The posterior probability of $R_{4}$ converges in approximately 1 second. However, $R_{4}$ is the preferred hypothesis as early as $0.2 \mathrm{~s}$. Thus, the MME algorithm correctly identifies the stator resistance when operating at rated speed and rated torque.

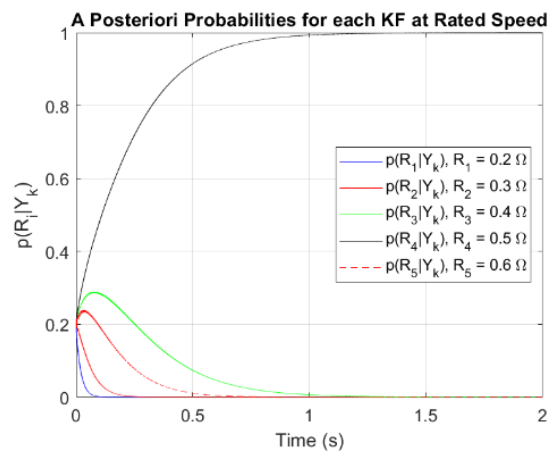

Fig. 3: Posterior probability of each hypothesis when the case study motor operates at rated speed. The estimated stator resistance is $0.5 \Omega$, and the true resistance is $0.49 \Omega$.

In Fig. 4, the estimate of $r_{s}$ from the EKF is presented. Note that the mean estimate is $1.5787 \Omega$. This is significantly larger $(224 \%)$ than the true stator resistance of $0.49 \Omega$. The error in the estimate may be a result of errors introduced by the Forward Euler discretization. Essentially, a sample period of $T=200 \mu$ s results in about 29 samples per fundamental electrical cycle. However, the back emf contains harmonics up to the 13th, and at this sampling period there are only two samples per 13th harmonic cycle. In Fig. 5, the estimate of $r_{s}$ from the FM-EKF is presented. The mean estimate is 1.5779 $\Omega$, which is significantly larger (222\%) than the true value of $0.49 \Omega$ and only a minimal improvement over the estimate from the EKF. 


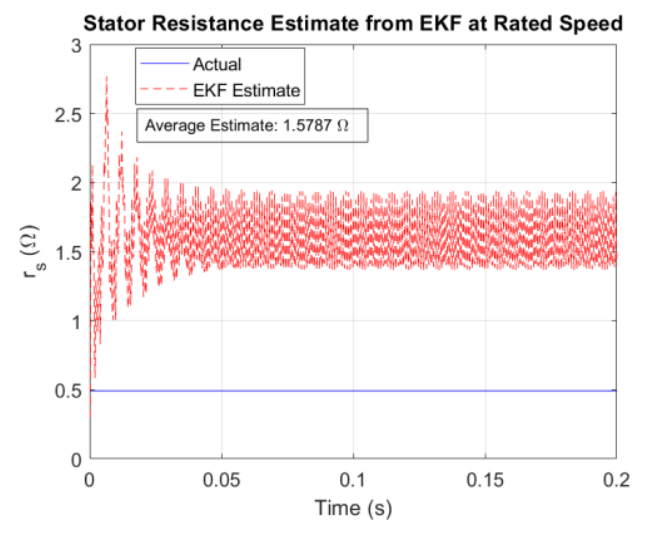

Fig. 4: Stator resistance estimate produced by the EKF at rated speed. The average estimated stator resistance is $1.5787 \Omega$, and the true resistance is $0.49 \Omega$.

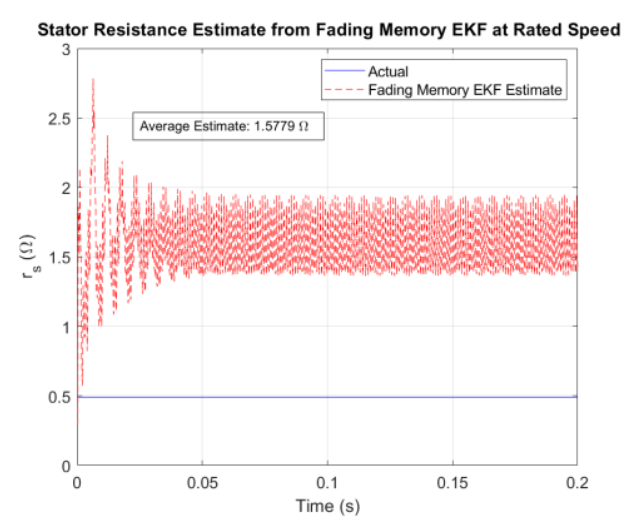

Fig. 5: Stator resistance estimate produced by the FM-EKF at rated speed. The average estimated stator resistance is 1.5779 $\Omega$, and the true resistance is $0.49 \Omega$.

\subsection{Results at One-Half Rated Speed}

In Fig. 6, the posterior probabilities of the five hypotheses of the MME algorithm are presented as a function of time. The posterior probability of $R_{4}=0.5 \Omega$ converges to 1 in about 1 second and again appears to be the preferred hypothesis at $0.2 \mathrm{~s}$. Thus, the MME algorithm correctly identifies the stator resistance when operating at one-half rated speed and rated torque.

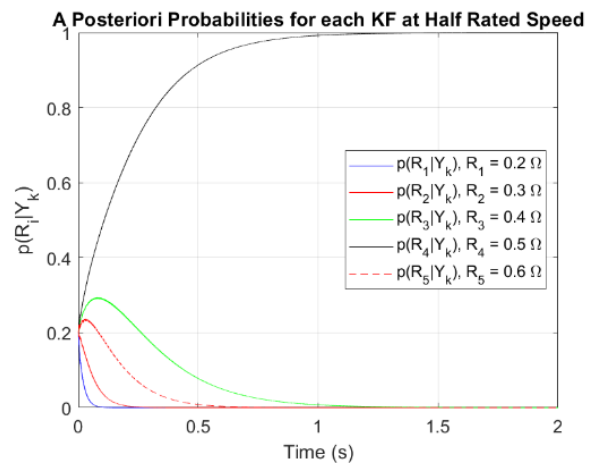

Fig. 6: Posterior probability of each hypothesis when the case study motor operates at one-half rated speed. The estimated stator resistance is $0.5 \Omega$, and the true resistance is $0.49 \Omega$.

In Fig. 7, the estimate of $r_{s}$ from the EKF is presented. At one-half rated speed, the mean estimate is $0.7508 \Omega$. This is significantly larger (53.2\%) than the true stator resistance of $0.49 \Omega$, but it is an improvement over the estimate from the EKF at rated speed. At one-half rated speed, the number of samples per electrical cycle increases to approximately 58 samples per fundamental electrical cycle, and 4.5 samples per 13th harmonic cycle. This indicates the estimate from the EKF improves as the motor speed decreases. In Fig. 8, the estimate of $r_{s}$ from the FM-EKF is presented. The mean estimate is $0.7505 \Omega$, which remains significantly larger $(53.2 \%)$ than the true value of $0.49 \Omega$ and indicates the FM-EKF is similarly affected by the sampling period. Note that at one-half rated speed, the FM-EKF does not significantly improve on the estimate from the EKF. 


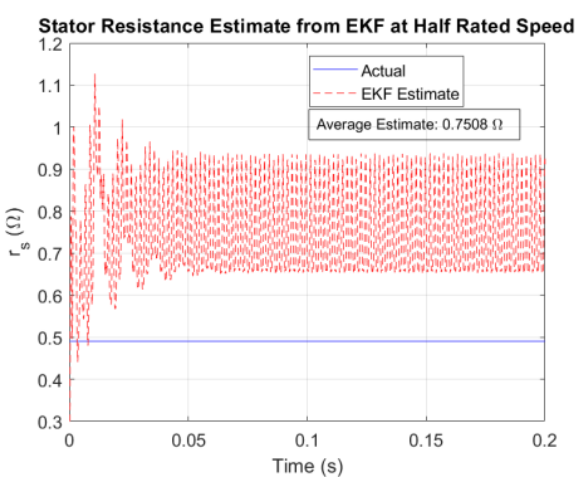

Fig. 7: Stator resistance estimate produced by the EKF at onehalf rated speed. The average estimated stator resistance is $0.7508 \Omega$, and the true resistance is $0.49 \Omega$.

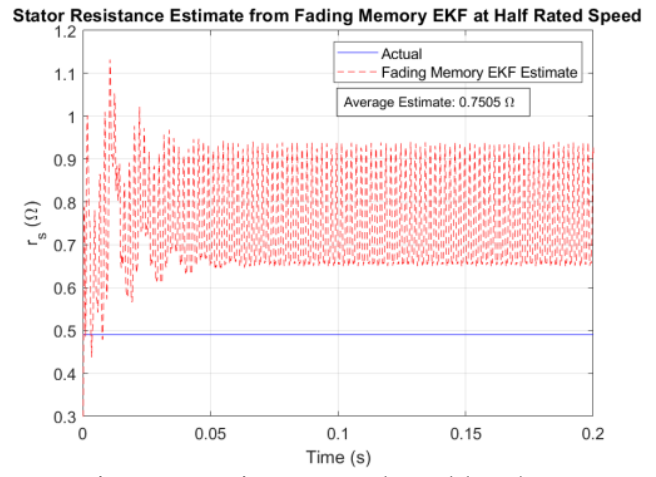

Fig. 8: Stator resistance estimate produced by the FM-EKF at one-half rated speed. The average estimated stator resistance is $0.7505 \Omega$, and the true resistance is $0.49 \Omega$.

\subsection{Results at One-Quarter Rated Speed}

In Fig. 9, the posterior probabilities of the five hypotheses of the MME algorithm are presented as a function of time. Again, the posterior probability of $R_{4}=0.5 \Omega$ converges to 1 in about 1 second, correctly identifying the parameter within the tolerance of the quantization.

In Fig. 10, the estimate of $r_{s}$ from the EKF is presented. At one-quarter rated speed, the mean estimate is $0.5526 \Omega$. This is $12.8 \%$ larger than the true stator resistance of $0.49 \Omega$. Note that the estimate does not improve at the same rate that the speed is decreased despite the increase to 116 samples per fundamental electrical cycle, and 9 samples per 13th harmonic cycle. In Fig. 11, the estimate of $r_{s}$ from the FM-EKF is presented. The mean estimate is $0.5525 \Omega$, which remains $12.8 \%$ larger than the true value of $0.49 \Omega$. By one-quarter rated speed, the FM-EKF does not improve on the estimate from the EKF, indicating sampling period and discretization method have a greater effect on accurate estimation of $r_{s}$.

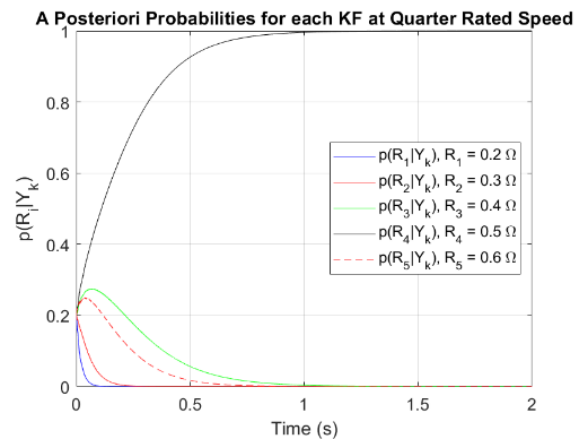

Fig. 9: Posterior probability of each hypothesis when the case study motor operates at one-quarter rated speed. The estimated stator resistance is $0.5 \Omega$, and the true resistance is $0.49 \Omega$. 


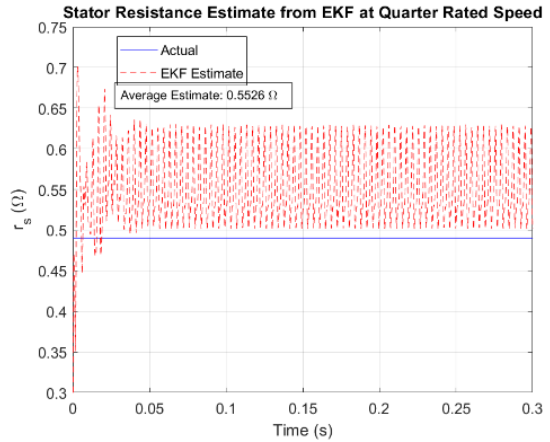

Fig. 10: Stator resistance estimate produced by the EKF at onequarter rated speed. The average estimated stator resistance is $0.5526 \Omega$, and the true resistance is $0.49 \Omega$.

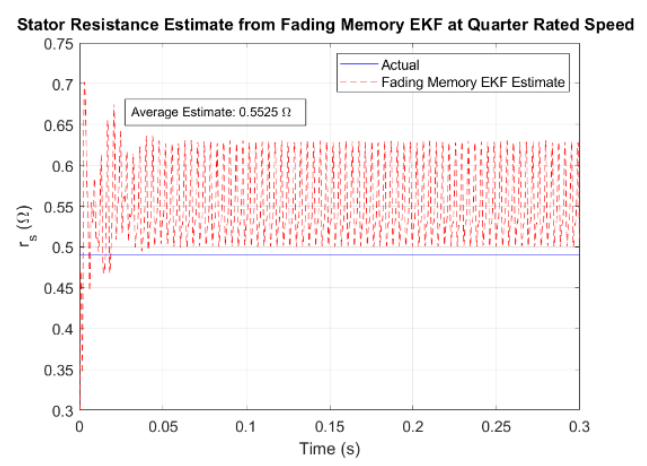

Fig. 11: Stator resistance estimate produced by the FM-EKF at one-quarter rated speed. The average estimated stator resistance is $0.5525 \Omega$, and the true resistance is $0.49 \Omega$.

\section{Conclusions}

The results from each of the estimators are summarized in Table 2. The MME algorithm utilizing Kalman filters identified the stator resistance within the tolerance of the quantized parameter space during operation at all three speeds. In contrast, the EKF cannot identify the parameter, though the estimates improve as the operating speed decreases. This indicates that a sampling period of $T=200 \mu \mathrm{s}$ is not sufficiently small to capture the system dynamics. Furthermore, the sampling period may not be sufficiently reduced to be within the region of numerical stability of the Forward Euler method. Thus, it would be more effective to improve the discretization of the system. Additionally, the FM-EKF provides minimal improvement over the EKF, particularly at lower operating speeds. This suggests that improving the discretization of the system would result in a greater improvement than the fading memory modification.

Table 2: Summary of Stator Resistance Estimates.

\begin{tabular}{|c|c|c|c|}
\hline & MME with KF & EKF & FM-EKF \\
\hline $100 \%$ Speed & $0.5 \Omega$ & $1.5787 \Omega$ & $1.5779 \Omega$ \\
\hline $50 \%$ Speed & $0.5 \Omega$ & $0.7508 \Omega$ & $0.7505 \Omega$ \\
\hline $25 \%$ Speed & $0.5 \Omega$ & $0.5526 \Omega$ & $0.5525 \Omega$ \\
\hline
\end{tabular}

Since implicit methods are challenging to implement on a nonlinear system such as in the parameter identification problem, it is highly desirable to preserve the linearity of the original system if the system is discretized. This highlights one major advantage of the MME algorithm over other parameter identification techniques. Future work includes applying these three estimators to the stator resistance estimation problem when pulse-width modulation terminal excitation is used.

\section{Appendix}

Consider a linear time varying stochastic system

$$
\begin{gathered}
x_{k+1}=A_{k} x_{k}+B_{k} u_{k}+F_{k} v_{k} \\
y_{k}=C_{k} x_{k}+D_{k} u_{k}+G_{k} w_{k} \\
{\left[\begin{array}{l}
x_{0} \\
v_{k} \\
w_{k}
\end{array}\right] \sim N\left(\left[\begin{array}{c}
\bar{x}_{0} \\
0 \\
0
\end{array}\right],\left[\begin{array}{ccc}
X_{0} & 0 & 0 \\
0 & V_{k} & 0 \\
0 & 0 & W_{k}
\end{array}\right]\right)}
\end{gathered}
$$

The Kalman filter for (18) is expressed by (20) The predict and update equations are combined in this formulation.

$$
\hat{x}_{k+1}=A_{k} \hat{x}_{k}+B_{k} u_{k}+K_{k}\left(y_{k}-C_{k} \hat{x}_{k}-D_{k} u_{k}\right)
$$

The Kalman gain $K_{k}$ is defined as

$$
K_{k}=A_{k} P_{k} C_{k}^{T}\left(C_{k} P_{k} C_{k}^{T}+G_{k} W_{k} G_{k}^{T}\right)^{-1}
$$

The covariance of the state estimate $P_{k}$ is calculated as

$$
P_{k+1}=A_{k} P_{k} A_{k}^{T}+F_{k} V_{k} F_{k}^{T}-A_{k} P_{k} C_{k}^{T}\left(C_{k} P_{k} C_{k}^{T}+G_{k} W_{k} G_{k}^{T}\right)^{-1} C_{k} P_{k} A_{k}^{T}
$$




\section{References}

[1] H. A. Toliyat, E. Levi and M. Raina, "A review of RFO induction motor parameter estimation techniques," IEEE Transactions on Energy Conversion, vol. 18, no. 2, pp. 271-283, 2003.

[2] T. Noguchi, S. Kondo and I. Takahashi, "Field-oriented control of an induction motor with robust on-line tuning of its parameters," IEEE Transactions on Industry Applications, vol. 33, no. 1, pp. 35-42, 1997.

[3] B. M. Chandra, B. V. Prasantlr and K. G. Manindher, "A Review on Parameter Estimation Techniques in Decoupling Control of Induction Motor Drive," in 2018 4th International Conference on Electrical Energy Systems (ICEES), 2018.

[4] J. L. Zamora and A. Garcia-Cerrada, "Online estimation of the stator parameters in an induction motor using only voltage and current measurements," IEEE Transactions on Industry Applications, vol. 36, no. 3, pp. 805-816, 2000.

[5] Y. Sangsefidi, S. Ziaeinejad, A. Mehrizi-Sani, H. Pairodin-Nabi and A. Shoulaie, "Estimation of Stator Resistance in Direct Torque Control Synchronous Motor Drives," IEEE Transactions on Energy Conversion, vol. 30, no. 2, pp. 626634, 2015.

[6] F. Baneira, L. Asiminoaei, J. Doval-Gandoy, H. A. M. Delpino, A. G. Yepes and J. Godbersen, "Estimation Method of Stator Winding Resistance for Induction Motor Drives Based on DC-Signal Injection Suitable for Low Inertia," IEEE Transactions on Power Electronics, vol. 34, no. 6, pp. 5646-5654, 2019.

[7] A. R. Strandt, A. P. Strandt, S. C. Schneider and E. E. Yaz, "Stator Resistance Estimation Using Adaptive Estimation via a Bank of Kalman Filters," in 2018 Annual American Control Conference (ACC), Milwaukee, 2018.

[8] P. Pillay and R. Krishnan, "Modeling of permanent magnet motor drives," IEEE Transactions on Industrial Electronics, vol. 35, no. 4, pp. 537-541, 1988.

[9] S. D. Wilson, G. W. Jewell and P. G. Stewart, "Resistance estimation for temperature determination in PMSMs through signal injection," in 2005 IEEE International Conference on Electric Machines and Drives, 2005.

[10] B. D. O. Anderson and J. B. Moore, Optimal Filtering, Mineola, NY: Dover Publications, Inc., 2005.

[11] D. Simon, Optimal State Estimation: Kalman, H-Infinity, and Nonlinear Approaches, Hoboken, NJ: John Wiley and Sons, Inc., 2006.

[12] R. F. Stengel, in Optimal Control and Estimation, Mineola, NY, Dover Publications, Inc., 1994, pp. 402 - 407. 